Nathália Gonçalves de Moura;

Nághela Gonçalves de Moura;

Adriana Ferreira de Carvalho;

Verônica Nogueira do Nascimento

\title{
Políticas Públicas de, com, para pessoas com deficiência
}

\author{
Public policies of, with, to disabled people
}

Nathália Gonçalves de Moura a

Nághela Gonçalves de Moura b

Adriana Ferreira de Carvalho ${ }^{c}$

Verônica Nogueira do Nascimento ${ }^{d}$

a Bacharela em Fisioterapia pela Faculdade Leão Sampaio (2014)

Especialista em Docência do Ensino Superior pelo Centro Universitário Doutor Leão Sampaio - UniLeão (2016). E-mail: nathaliagdm@hotmail.com

b Bacharela em Direito pela Faculdade Paraíso do Ceará - FAP CE (2014) Mestra em Desenvolvimento Regional Sustentável pela Universidade Federal do Cariri - UFCA (2017). E-mail: naghela.gm@gmail.com

c Faculdade de Medicina de Juazeiro do Norte, Departamento de Pediatria. Graduada em Medicina pela Universidade Federal do Pernambuco - UFPE (2002); Residência médica em Pediatria pela Universidade Federal do Pernambuco - UFPE (2005); Residência médica em Neonatologia pela Universidade Federal do Pernambuco - UFPE (2007); Mestra em Desenvolvimento Regional Sustentável pela Universidade Federal do Cariri - UFCA (2017). E-mail: adricarv02@hotmail.com

d Universidade Estadual Vale do Acaraú, Unidade Cariri Bacharela em Enfermagem pela Faculdade Leão Sampaio (2010)

Especialista em Saúde da Família pela Universidade Estadual Vale do Acaraú - UVA (2010). Especialista em Docência do Ensino Superior pelo Centro Universitário Doutor Leão Sampaio - UniLeão (2012).

Mestra em Desenvolvimento Regional Sustentável pela Universidade Federal do Cariri - UFCA (2017). E-mail: veronykka@gmail.com

Recebido em: 15/12/2017 I Aceito em: 25/06/2018 
Nathália Gonçalves de Moura;

Nághela Gonçalves de Moura;

Adriana Ferreira de Carvalho;

Verônica Nogueira do Nascimento

\title{
RESUMO
}

Com a promulgação da Constituição de 1988, o tema da marginalização das pessoas com deficiência passou a ser enfrentado como uma demanda a ser respondida pelo Estado, restando claro a opção do legislador constituinte pela abordagem estadocêntrica no que toca a elaboração de políticas públicas para este segmento populacional. Entretanto, para implementar políticas públicas inclusivas, um forte comprometimento e ações de uma série de atores não estatais são necessários. Este ensaio teórico objetivou redimensionar o papel dos atores estatais, da sociedade em geral e organizada e das próprias pessoas com deficiência e suas entidades representativas, no enfrentamento do problema público da marginalização dos sujeitos com deficiência. Com a ratificação da Convenção Sobre os Direitos das Pessoas com Deficiência e ao assumir o compromisso de atingir até 2030 os Objetivos de Desenvolvimento do Milênio, a sociedade e as próprias pessoas com deficiência também foram conclamadas a participar da elaboração de (e até mesmo desenvolver) políticas públicas com vistas a garantir os direitos dos sujeitos com deficiência. A efetivação de políticas públicas inclusivas apresentou-se como uma tarefa de toda a sociedade brasileira e não apenas dos governos, exigindo o empenho, entre outros, dos setores privados, das instituições de ensino, das comunidades e o engajamento de cada cidadão, com e sem deficiências. Tudo com o propósito de promover a equiparação de oportunidades e a realização de sonhos e projetos por parte dos brasileiros com deficiência.

Palavras-chave: Sociedade. Estado. Pessoas com deficiência. Participação. Políticas públicas inclusivas.

\begin{abstract}
After the promulgation of the 1988 Constitution, the subject of the marginalization of disability people have come to be faced as a demand to be answered by the State, leaving the option of the constituent legislator for the state-centered approach regarding the elaboration of public policies for this segment populational. Yet, to implement inclusive public policies, a strong commitment and actions of a range of non-state agents are really necessary. This theoretical subject matter aimed redimensioning the participation of the states agents from the society in general and the organized society and the own disability people and their representative entities, in facing the public problem of the marginalization of disabled individuals. With the ratification of the Convention on People's rights with Disabilities and the commitment to reach the Millennium Development Goals by 2030, society and disabled people themselves have also been called upon to participate in the elaboration of public policies aiming at ensuring the individuals disability rights. The accomplishment of inclusive public policies presented itself as a task for all Brazilian society, and not only for governments, requiring the commitment, among others, of the private educational sectors, communities and the engagement of each citizen with disabilities or not. This proposal will be able to develop and promotes the equalization of opportunities and will achieve the dreams by helping Brazilians projects with disabilities.
\end{abstract}

Key words: Society. State. People with Disabilities. Participation. Inclusive public policies.

Ciência e Sustentabilidade - CeS / Juazeiro do Norte, v. 4, n. 1, p. 79-94, jan/jun - 2018 


\section{INTRODUÇÃO}

No Brasil, durante décadas, a temática da marginalização das pessoas com deficiência ficou sob a invisibilidade política e legal e as políticas e programas que promoviam minimamente os direitos dos sujeitos com deficiência encontravam-se no âmbito da caridade, do assistencialismo e dos cuidados familiares. Este largo período justifica-se tanto pelo fato de que o modelo médico preponderava como recurso explicativo da deficiência, quanto pelo fato de que a deficiência era compreendida como um fenômeno associado ao azar, ao divino ou a uma experiência privada sem a necessidade da intervenção estatal (SANTOS, 2008).

Foi com a Constituição Federal de 1988 (CF/88), que o tema começou a ser enfrentado como uma demanda social a ser respondida pelo Estado, que atraiu para si a responsabilidade de garantir a igualdade e a justiça às pessoas com deficiência (SANTOS, 2008). Em seu dispositivo 23, inciso II, a CF/88, apregoa que é competência comum da União, dos Estados, do Distrito Federal e dos Municípios cuidar da proteção e garantia das pessoas com deficiência (BRASIL, 1988).

Quando a atual Magna Carta decidiu, por meio do legislador constituinte, que cabe a todos os entes da federação "cuidar" das pessoas com deficiência, resta claro a opção pela abordagem estadocêntrica no que toca a elaboração de políticas públicas para este segmento populacional. Todavia, para implementar políticas públicas inclusivas, um forte comprometimento e ações de uma série de atores não estatais são necessários.

Diante do até aqui exposto, questiona-se: como o Estado, a sociedade e as próprias pessoas com deficiência podem desenvolver (ou influir em) políticas públicas voltadas para a inclusão política, social e econômica dos sujeitos com deficiência?

Para responder a este questionamento, foi realizado um ensaio teórico que tem como objetivo levantar uma discussão acerca da temática por meio da exposição das ideias e pontos de vista dos autores, tendo embasamento teórico na literatura científica existente e nos documentos e publicações do governo brasileiro (MARCONI, LAKATOS, 2003). 
Nathália Gonçalves de Moura;

Nághela Gonçalves de Moura;

Adriana Ferreira de Carvalho;

Verônica Nogueira do Nascimento

A presente pesquisa objetivou redimensionar o papel dos atores estatais, da sociedade em geral e organizada e das próprias pessoas com deficiência e suas entidades representativas, no enfrentamento do problema público da marginalização das pessoas com deficiência, como condição necessária para a elaboração, e efetivação exitosa de políticas públicas sustentáveis e inclusivas.

No tópico subsequente a esta introdução serão abordadas as diferentes formas pelas quais a sociedade civil organizada e as organizações de pessoas com deficiência podem influir no processo de elaboração de uma política pública, bem como destacar orientações de acessibilidade para assegurar a participação dos cidadãos com deficiência na tomada de decisões que lhes afetem. No item 3, serão elencados os diferentes modos pelos quais as organizações não governamentais, organizações privadas, organismos internacionais, redes de políticas públicas e as pessoas com deficiência podem atuar como protagonistas no desenho das políticas públicas. Em seguida, serão tecidas as considerações finais.

\section{POLÍTICAS PÚBLICAS SUSTENTÁVEIS E INCLUSIVAS}

A responsabilização do governo brasileiro frente as pessoas com deficiência se perfaz por meio de medidas denominadas de políticas públicas (TEIXEIRA, 2010). Uma política pública é uma diretriz materializada em decisões ou ações com intencionalidade pública e elaborada para em frentar um problema entendido como coletivamente relevante (SECCHI, 2013).

Consoante Werneck (2011), uma política pública inclusiva é aquela capaz de atender às necessidades de acessibilidade dos cidadãos em interação com seus territórios de origem, nos quais vivem e se relacionam, de modo presencial ou virtual, em infinito processo de adaptação e participação republicana.

O objetivo das políticas públicas inclusivas é promover a equiparação de oportunidades, para que a deficiência não seja utilizada como um fator limitador ou um impedimento à realização de sonhos e projetos, valorizando o protagonismo e as escolhas dos brasileiros com deficiência (BRASIL, 2013, TEIXEIRA, 2010).

Nas palavras de Secchi (2013) e Rua e Romanini (2013) as políticas públicas ganham corpo por meio de:

Ciência e Sustentabilidade - CeS / Juazeiro do Norte, v. 4, n. 1, p. 79-94, jan/jun - 2018 
a) Políticas, programas, planos e projetos: como por exemplo, Programa Brasil Acessível; e Plano Viver Sem Limites, dentre outros (BRASIL, 2006, BRASIL, 2011);

b) fornecimento de produtos e serviços: como por exemplo, o fornecimento de aparelhos auditivos, próteses, cadeiras de rodas, dentre outros;

c) Recursos financeiros: como por exemplo, o Benefício de Prestação Continuada (BRASIL, 1993);

d) Impostos e taxas: sendo um exemplo a redução de impostos para aquisição de veículos por pessoa com deficiência (BRASIL, 1998); e

e) Legislação: sendo um exemplo, a Lei Brasileira de Inclusão dentre outros (BRASIL, 2015).

Na prática, a realização dos direitos das pessoas com deficiência exige ações em ambas as frentes, a do direito universal e a do direito de grupos específicos. Sendo que esta última é denominada de ação afirmativa.

A ação afirmativa é aquela que, a partir do reconhecimento de uma iniquidade, cria políticas públicas específicas para modificar esse quadro, de modo a assegurar acesso a direitos, bens e serviços semelhantes ao remanescente da população (GRUPO DE ESTUDOS MULTIDISCIPLINARES DA AÇÃO AFIRMATIVA, 2011).

A ação afirmativa consagra o Princípio da Isonomia", o qual determina que se dê tratamento igual aos que se encontram em situação equivalente, e que se trate de maneira desigual os desiguais na medida de suas desigualdades (LENZA, 2012).

A Convenção Sobre os Direitos das Pessoas com Deficiência, ratificada pelo Brasil com status de Emenda Constitucional, preceitua que "as medidas específicas que forem necessárias para acelerar ou alcançar a efetiva igualdade das pessoas com deficiência, não serão consideradas discriminatórias (BRASIL, 2009, p. 6). Contudo, em um futuro que se deseja sustentável, bastará o uso da palavra pessoa, sem o incremento de qualquer adjetivo e bastará o emprego da concepção da transversalidade das questões atinentes as deficiências, sem a execução de políticas públicas específicas.

Assim, a transversalidade é um princípio, um comando orientador na elaboração das políticas públicas que objetivem alcançar a todos independentemente

Ciência e Sustentabilidade - CeS / Juazeiro do Norte, v. 4, n. 1, p. 79-94, jan/jun - 2018 
Nathália Gonçalves de Moura;

Nághela Gonçalves de Moura;

Adriana Ferreira de Carvalho;

Verônica Nogueira do Nascimento

de os sujeitos destinatários terem ou não uma restrição de funcionalidade nas estruturas do corpo ou um comprometimento cognitivo.

Neste sentido, uma política pública inclusiva não é necessariamente uma política pública exclusiva. Para que existam ganhos que favoreçam as pessoas com deficiência, a agenda de desenvolvimento precisa contemplar a perspectiva de impulsionar o desenvolvimento sustentável associando acessibilidade e inclusão, no entendimento de que deve ocorrer a incorporação desses conceitos como valor associado ao conceito de desenvolvimento (WERNECK, 2011). "Não são as soluções acessíveis e inclusivas que têm que ser sustentáveis, as soluções sustentáveis é que têm que ser acessíveis e inclusivas", complementa (2013, p. 7).

Em seu preâmbulo, alínea g, a Convenção Sobre os Direitos das Pessoas com Deficiência, assevera "[...] a importância de trazer questões relativas à deficiência ao centro das preocupações da sociedade como parte integrante das estratégias relevantes de desenvolvimento sustentável" (BRASIL, 2009, p. 1).

A Convenção sob análise oferece um marco para a construção de uma agenda de conjugação entre os direitos humanos das pessoas com deficiência e a do desenvolvimento sustentável. A expectativa é que as demandas das pessoas com deficiência estejam presentes nas reflexões sobre os caminhos que a humanidade pretende seguir rumo ao desenvolvimento sustentável (MOURA, 2017).

\section{INFLUIR EM POLÍTICAS PÚBLICAS}

A política pública depende de procedimentos e de processos institucionais governamentais, e embora não confira a atores não estatais o privilégio de estabelecer um processo de política pública, a concepção estadocentrica permite que atores não estatais até tenham influência no processo de feitura e execução de políticas públicas (SECCHI, 2013).

O papel da sociedade civil organizada deve consolidar-se em todos os níveis, mediante uma ampla participação nas decisões que lhes afetem. Para tanto, "Os governos devem oferecer condições para facilitar o direito de todos os estratos sociais de ter voz e ocupar um papel ativo na construção de um futuro sustentável" (CAMARGO, 2002, p. 107).

Ciência e Sustentabilidade - CeS / Juazeiro do Norte, v. 4, n. 1, p. 79-94, jan/jun - 2018 
A participação popular pode acontecer tanto no âmbito da sociedade como na esfera do Estado (BRASIL, 2007, p. 49)

\begin{abstract}
No primeiro caso, organiza-se em torno de entidades ou organizações totalmente independentes do poder público e não organizadas por iniciativa governamental. [...] $\mathrm{Na}$ outra ponta, encontram-se espaços criados pelo próprio Estado, institucionalizados ou não, com origem na luta autônoma dos movimentos sociais ou por iniciativa do próprio governo, pretendendo criar canais de relacionamento direto entre o Estado e a sociedade.
\end{abstract}

As organizações da sociedade civil têm uma gama de finalidades (educacional, cultural, de promoção da saúde, da inclusão, da cidadania, dentre outras), que em seu conjunto, colaboram para aumentar a habilidade da sociedade de exercer a sua cidadania e desenvolver-se de modo sustentável (INSTITUTO BRASILEIRO DE GEOGRAFIA E ESTATÍSTICA, 2012).

\title{
3.1 "Nada Sobre Nós, Sem Nós"
}

O lema das pessoas com deficiência, "Nada sobre nós, sem nós", nasceu da proximidade histórica do movimento negro com o de pessoas com deficiência. William Rowland, em seu artigo "Nada Sobre Nós, Sem Nós: Algumas Reflexões Sobre o Movimento das Pessoas com Deficiência na África do Sul”, registrou que, em 1981, o governo local se recusou a reconhecer o ano internacional das pessoas com deficiência (SASSAKI, 2007).

Em 1986, às escondidas das próprias pessoas com deficiência, o governo declarou convenientemente o "Ano das Pessoas Deficientes da África do Sul". Na ocasião, as pessoas com deficiência se retiraram da conferência e só retornaram quando a palavra lhes foi permitida, por meio da leitura de um catálogo de injustiças, contendo a dupla discriminação gerada pelo apartheid e pelas deficiências (SASSAKI, 2007).

QUADRO 1- Explicativo do lema !Nada sobre nós, sem nós"

\begin{tabular}{|c|l|}
\hline \multirow{2}{*}{ Nada } & $\begin{array}{l}\text { Nenhum resultado: lei, política pública, programa, } \\
\text { serviço, projeto, campanha, financiamento, } \\
\text { edificação, aparelho, equipamento, utensílio, } \\
\text { sistema, estratégia, benefício etc. Cada um destes }\end{array}$ \\
\hline
\end{tabular}

Ciência e Sustentabilidade - CeS / Juazeiro do Norte, v. 4, n. 1, p. 79-94, jan/jun - 2018 
Nathália Gonçalves de Moura;

Nághela Gonçalves de Moura;

Adriana Ferreira de Carvalho;

Verônica Nogueira do Nascimento

\begin{tabular}{|c|l|}
\hline & \begin{tabular}{l} 
resultados se localiza em um dos (em mais de um \\
ou todos os) campos de atividade como, por \\
exemplo, educação, trabalho, saúde, reabilitação, \\
transporte, lazer, recreação, esportes, turismo, \\
cultura, artes, religião. \\
\hline "a respeito das pessoas com deficiência". Essas \\
pessoas são de qualquer etnia, raça, gênero, \\
idade, nacionalidade, naturalidade etc., e a \\
deficiência pode ser física, intelectual, visual, \\
auditiva, psicossocial ou múltipla.
\end{tabular} \\
\hline , (vírgula) & $\begin{array}{l}\text { Segue-se uma vírgula (com função de elipse, uma } \\
\text { figura de linguagem que substitui uma locução } \\
\text { verbal) que, neste caso, substitui a expressão } \\
\text { "haverá de ser gerado". }\end{array}$ \\
\hline Sem Nós & $\begin{array}{l}\text { "Sem a plena participação das próprias pessoas } \\
\text { com deficiência". Esta participação, individual ou } \\
\text { coletiva, mediante qualquer meio de comunicação, } \\
\text { deverá ocorrer em todas as etapas do processo de } \\
\text { geração dos resultados acima referidos. As } \\
\text { principais etapas são: a elaboração, o refinamento, } \\
\text { a implementação, o monitoramento, a avaliação e } \\
\text { o contínuo aperfeiçoamento. }\end{array}$ \\
\hline
\end{tabular}

Fonte: Sassaki $(2007$, p. 1)

Nota: Compilação das autoras.

Geralmente as pessoas com deficiência possuem uma visão particular de sua condição e de sua situação. Na elaboração e execução de políticas, leis e serviços, as pessoas com deficiência devem ser consultadas e envolvidas ativamente. Nesta direção, o Relatório Mundial Sobre a Deficiência (ORGANIZAÇÃO MUNDIAL DA SAÚDE, 2012, p. 19), versa que:

As pessoas com deficiência possuem o controle sobre suas vidas e, portanto, precisam ser consultadas sobre as ações que lhes dizem respeito diretamente - seja na saúde, na educação, na reabilitação ou na vida em comunidade. $O$ apoio no processo de decisão pode ser necessário para permitir que os indivíduos comuniquem suas necessidades e escolhas.

Neste sentido, o documento final da Rio+20, "O Futuro que Queremos", reconhece a importância da ampla participação Das pessoas com deficiência nos

Ciência e Sustentabilidade - CeS / Juazeiro do Norte, v. 4, n. 1, p. 79-94, jan/jun - 2018 
processos que contribuem para a tomada de decisão, planejamento e implementação de políticas e programas para o desenvolvimento sustentável em todos os níveis (UNITED NATIONS, 2012).

Assim, em todos os âmbitos de tomada de decisões, os governos precisam criar ou consolidar mecanismos regulares para consulta e diálogo que viabilizem às pessoas com deficiência, mediante suas entidades representativas, contribuir para a elaboração, execução, monitoramento e avaliação de todas as políticas, programas e ações (SASSAKI, 2007).

As diferentes estratégias de influência em políticas públicas, ou de co-gestão pública, são:

f) Se utilizar das representações, como os conselhos municipal, estadual e nacional dos direitos da pessoa com deficiência, "para promover a participação ativa da sociedade civil em instâncias decisórias ou em canais de diálogo com - governo, democratizando a tomada de decisão sobre a vida coletiva" (GHANEM, 2007, p. 33);

g) Fazer pressão (lobby) sobre os gestores, em todos os níveis de governo, mobilizando-os em torno de ações como mudanças da legislação, das políticas públicas e das decisões orçamentárias que traduzam a real necessidade das pessoas com deficiência, "ampliando o conceito de justiça social em nosso país e garantindo direitos e deveres para todos(as)" (GHANEM, 2007, p. 33);

h) Implementar experiências exemplares e inspiradoras na esfera privada, para que estas sejam assumidas e multiplicadas, mesmo que parcialmente, pelo poder público (GHANEM, 2007); e

i) Prestar serviços de natureza especializada a governos, influenciando o desenho das políticas públicas em práticas cotidianas (GHANEM, 2007).

A nova agenda de desenvolvimento sustentável, a ser implementada por todos os países do mundo durante os próximos 15 anos, até 2030, é formada por 17 Objetivos de Desenvolvimento Sustentável, dentre os quais, o objetivo 16, meta 7 que consiste em assegurar a tomada de decisão responsável, inclusiva, participativa e representativa em todos os níveis (NACIONES UNIDAS, 2015). 
Nathália Gonçalves de Moura;

Nághela Gonçalves de Moura;

Adriana Ferreira de Carvalho;

Verônica Nogueira do Nascimento

O sujeito coletivo, materializado nos segmentos organizados da sociedade ou nos movimentos sociais, pode e deve participar da reinvenção do Estado e influir na elaboração das políticas públicas. Para tanto, os encontros para discussão devem ser realizados em espaços arquitetonicamente acessíveis e deve ser providenciada a eliminação de barreiras nas comunicações, mediante a presença do tradutor/intérprete da Língua Brasileira de Sinais e da disponibilização de materiais em formatos alternativos, tais como tinta, braile, áudio ou digital.

É forçoso reconhecer que esta participação não deve ser mitigada ao mero recebimento de informações ou ao endosso de decisões. As pessoas com deficiência devem ter vez e voz ativa e seu brado deve ser levado em conta.

Quando as pessoas com deficiência tem assegurada sua participação nos processos decisórios de seu país, Estado ou município, relatando suas necessidades, a tendência é que as políticas públicas sejam implementadas não só de modo inclusivo, mas também e consequentemente, sustentável.

\section{NOVOS ATORES EM CENA}

Durante Séculos, a deficiência foi entendida como uma expressão do azar, um drama pessoal e uma experiência enfrentada na esfera privada, sob a tutela dos cuidados familiares e das instituições filantrópicas. Isto porque, a deficiência era compreendida como um fenômeno na esfera da patologia ou da anormalidade, denominado de modelo médico da deficiência (SANTOS, 2008, MOURA, 2017).

A Convenção Sobre os Direitos das Pessoas com Deficiência, em seu preâmbulo, alínea e, apregoa que a deficiência é um conceito em evolução e que a deficiência "resulta da interação entre pessoas com deficiência e as barreiras devidas às atitudes e ao ambiente que impedem a plena e efetiva participação dessas pessoas na sociedade em igualdade de oportunidades com as demais pessoas" (BRASIL, 2009).

Além de atribuir não só ao Estado, mas também à sociedade civil o "cuidado" com este grupo, a Convenção inaugura uma oportunidade inédita de resgatar a abordagem multicêntrica do conceito de política pública (BRASIL, 2009), em que

Ciência e Sustentabilidade - CeS / Juazeiro do Norte, v. 4, n. 1, p. 79-94, jan/jun - 2018 
"Estado e sociedade se articulam em estruturas espontâneas e horizontais para a solução de problemas públicos" (SECCHI, 2013).

Estudiosos da abordagem multicêntrica atribuem o caráter "público" a uma política quando o problema que se tenta responder é público, e não um problema político, independentemente do ator responsável pertencer ou não ao aparelho estatal (SECCHI, 2013, RUA, ROMANINI, 2013).

A vertente multicêntrica considera organizações não governamentais, organizações privadas, organismos internacionais, redes de políticas públicas, juntamente com os agentes estatais, protagonistas no desenho das políticas públicas (SECCHI, 2013, RUA, ROMANINI, 2013).

Ainda que os governos possuam o papel mais expressivo, outros atores também desempenham papeis importantes no enfrentamento do problema público da marginalização das pessoas com deficiência. O Relatório Mundial Sobre a Deficiência (ORGANIZAÇÃO MUNDIAL DA SAÚDE, 2012, p. 23) sublinha algumas dessas medidas que as várias partes interessadas podem adotar:

\section{O setor privado pode:}

Facilitar o emprego das pessoas com deficiência, assegurando que 0 recrutamento é equitativo, que adaptações razoáveis são oferecidas, e que os empregados que se tornem deficientes serão apoiados no retorno ao trabalho.

Remover as barreiras de acesso ao microfinanciamento, de modo que as pessoas com deficiência possam desenvolver seus próprios negócios.

Desenvolver uma série de serviços de suporte de qualidade para pessoas com deficiência e suas famílias nos diferentes estágios do ciclo da vida.

Assegurar que projetos de construção, tais como edifícios públicos, escritórios e habitações incluam o acesso adequado as pessoas com deficiência.

Garantir que os produtos, sistemas e serviços relacionados as tecnologias de Informação e comunicação são acessíveis as pessoas com deficiência.

As instituições acadêmicas podem:

Remover as barreiras ao recrutamento e participação de estudantes e funcionários com deficiência;

Assegurar que cursos de formação profissional incluem adequadamente informações Sobre a deficiência, baseado em princípios de direitos humanos. Realizar pesquisas sobre as vidas das pessoas com deficiência e sobre as barreiras incapacitantes, em consulta com organizações de pessoas com deficiência.

As comunidades podem:

Desafiar e mudar suas próprias crenças e atitudes.

Promover a inclusão e participação das pessoas com deficiência em sua comunidade.

Assegurar que os ambientes comunitários são acessíveis as pessoas com deficiência, incluindo escolas, áreas de lazer e instalações culturais.

Desafiar o bullying e a violência contra as pessoas com deficiência.

Ciência e Sustentabilidade - CeS / Juazeiro do Norte, v. 4, n. 1, p. 79-94, jan/jun - 2018 
Nathália Gonçalves de Moura;

Nághela Gonçalves de Moura;

Adriana Ferreira de Carvalho;

Verônica Nogueira do Nascimento

Segundo Boff (1999, p. 6), "A libertação dos oprimidos deverá provir deles mesmos, na medida em que se conscientizam da injustiça de sua situação, se organizam entre si e começam com práticas que visam transformar estruturalmente as relações sociais iníquas". Neste sentido, O Relatório Mundial Sobre a Deficiência (ORGANIZAÇÃO MUNDIAL DA SAÚDE, 2012, p. 22), também chama a atenção das pessoas com deficiência em sua dimensão organizada. A publicação assinala que, as associações de pessoas com deficiência podem participar na vida pública por meio das seguintes iniciativas:

Apoiar a conscientização das pessoas com deficiência sobre seus direitos, a vida independente e o desenvolvimento de suas habilidades.

Apoiar as crianças com deficiência e suas famílias para assegurar a inclusão na educação.

Representar as perspectivas de seus membros junto àqueles responsáveis pelas decisões e aos prestadores de serviços em nível internacional, nacional e local, e defender seus direitos.

Contribuir para a avaliação e monitoramento dos serviços, e colaborar com os pesquisadores para apoiar pesquisas aplicadas que podem contribuir para o desenvolvimento dos serviços.

Promover a conscientização e a compreensão da opinião pública sobre os direitos das pessoas com deficiência - por exemplo, por meio de campanhas e iniciativas de formação sobre a igualdade de direitos das pessoas com deficiência.

Conduzir auditorias em ambientes, transportes, e outros sistemas e serviços de modo a promover a redução de barreiras.

Nos termos do Relatório Mundial Sobre a Deficiência (ORGANIZAÇÃO MUNDIAL DA SAÚDE, 2012, p. 23), as próprias pessoas com deficiência, mesmo que em uma atuação individualizada, podem:

\footnotetext{
Apoiar outras pessoas com deficiência por meio do apoio, do treinamento, da informação e do aconselhamento mútuos.

Promover os direitos das pessoas com deficiência no âmbito de suas comunidades.

Envolver-se nas campanhas de conscientização pública e marketing social. Participar de fóruns (internacionais, nacionais e locais) para determinar as prioridades de mudança, para influenciar a política e para moldar a prestação de serviços.

Participar em projetos de pesquisa.
}

É importante salientar que, mesmo não atuando de modo organizado ou nos movimentos sociais, a pessoa com deficiência pode e deve reivindicar sua dimensão de sujeito, recusando-se a permanecer nos lugares aprioristicamente determinados

Ciência e Sustentabilidade - CeS / Juazeiro do Norte, v. 4, n. 1, p. 79-94, jan/jun - 2018 
pela família, pela classe social, pelo lugar de origem ou por qualquer outra instituição socializadora.

\section{CONSIDERAÇÕES FINAIS}

A responsabilização do governo brasileiro frente aos cidadãos com deficiência operou-se com a promulgação da Constituição Federal de 1988 e se perfaz mediante a execução de políticas públicas.

A Convenção Sobre os Direitos das Pessoas com Deficiência atribuiu também à sociedade a responsabilidade por enfrentar a deficiência como uma questão social na esfera pública, promovendo a garantia dos direitos de quem a experimenta, nomeadamente a necessidade de adequações dos ambientes (em seus componentes físicos e humanos) às diversidades corporais (BRASIL, 2009).

Percebe-se que com o advento da Convenção, além do Estado, a sociedade também é chamada para remover as barreiras que dificultam ou até mesmo inviabilizam a plena participação das pessoas com deficiência na vida comunitária. A remoção destes obstáculos abrange posturas que vão desde o respeito às diferenças, até a efetivação de direitos, como à educação, ao trabalho, ao lazer e à cultura.

O Documento O Futuro que Queremos (UNITED NATIONS, 2012) e os Objetivos de Desenvolvimento do Milênio (NACIONES UNIDAS, 2015) reconhecem a importância de garantir, nos processos de elaboração, implementação e avaliação das políticas públicas, a ampla participação das pessoas com deficiência, uma vez que ninguém conhece tão profundamente suas realidades além delas mesmas (ORGANIZAÇÃO MUNDIAL DA SAÚDE, 2012).

A efetivação de políticas públicas inclusivas é, por tanto, uma tarefa para toda a sociedade brasileira e não somente para os governos. Exige o empenho, entre outros, dos setores privados, das instituições de ensino, das comunidades, como também o engajamento de cada cidadão, com e sem deficiências. Tudo com 0 propósito de promover a equiparação de oportunidades para que a deficiência não seja utilizada como um fator limitador ou um impedimento à realização de sonhos e projetos, valorizando o protagonismo e as escolhas dos brasileiros com deficiência. 
Nathália Gonçalves de Moura; Nághela Gonçalves de Moura; Adriana Ferreira de Carvalho; Verônica Nogueira do Nascimento

\section{REFERÊNCIAS}

BOFF, Leonardo. Saber cuidar: ética do humano. Rio de Janeiro: Vozes. 1999.

BRASIL. Presidência da República. Casa Civil. Subchefia para Assuntos Jurídicos. Constituição da República Federativa do Brasil de 1988. Brasília (DF), 1988. Disponível em: <https://www.planalto.gov.br/ccivil 03/constituicao/constituicao.htm>. Acesso em: 13 ago. 2017.

. Presidência da República. Casa Civil. Subchefia para Assuntos Jurídicos. Lei № 8.742 , de 7 de dezembro de 1993. Dispõe sobre a organização da Assistência Social e dá outras providências. Brasília (DF). 1993. Disponível em: $<$ http://www2.camara.leg.br/legin/fed/lei/1993/lei-8742-7-dezembro-1993-363163publicacaooriginal-1-pl.html>. Acesso em: 20 jun. 2016.

. Presidência da República. Casa Civil. Subchefia para Assuntos Jurídicos. Lei no 8.989, de 24 de fevereiro de 1995. Dispõe sobre isenção do Imposto sobre Produtos Industrializados (IPI) na aquisição de automóveis para utilização no transporte autônomo de passageiros, bem como por pessoas portadoras de deficiência física e aos destinados ao transporte escolar, e dá outras providências. Brasília (DF), 1995. Disponível em: <http://www2.camara.leg.br/legin/fed/lei/1995/lei8989-24-fevereiro-1995-349817-publicacaooriginal-1-pl.html>. Acesso em: 02 abr. 2016.

. Ministério das Cidades. Secretaria Nacional de Transporte e da Mobilidade Urbana. Construindo a cidade acessível. 1 ed. Brasília - DF, 2006. Disponível em: <http://www.fcee.sc.gov.br/index.php/informacoes/bibliotecavirtual/acessibilidade>. Acesso em: 8 nov. 2017.

. Ministério das Cidades. Caderno de Referência para Elaboração de Plano de Mobilidade Urbana. 2007. Disponível em: $<$ http://www.cidades.gov.br/images/stories/ArquivosSEMOB/Biblioteca/LivroPlanoMo bilidade.pdf $>$. Acesso em: 15 ago. 2015

. Presidência da República. Casa Civil. Subchefia para Assuntos Jurídicos Decreto № 6.949, de 25 de agosto de 2009. Promulga a Convenção Internacional sobre os Direitos das Pessoas com Deficiência e seu Protocolo Facultativo, Assinados em Nova York, em 30 de março de 2007. Brasília (DF), 2009. Disponível em: <http://www.planalto.gov.br/ccivil 03/ ato2007-2010/2009/decreto/d6949.htm>. Acesso em: 08 set. 2017.

. Presidência da República. Casa Civil. Subchefia para Assuntos Jurídicos. Decreto no 7.612, de 17 de novembro de 2011. Institui o Plano Nacional dos Direitos da Pessoa com Deficiência - Plano Viver sem Limite. Brasília - DF, 2011. Disponível em: <http://www2.camara.leg.br/legin/fed/decret/2011/decreto-7612-17-novembro2011-611789-publicacaooriginal-134271-pe.html>. Acesso em: 02 abr. 2016.

Ciência e Sustentabilidade - CeS / Juazeiro do Norte, v. 4, n. 1, p. 79-94, jan/jun - 2018 
. Secretaria de Direitos Humanos da Presidência da República, Secretaria Nacional de Promoção dos Direitos da Pessoa com Deficiência. Deficiência, viver sem limite - Plano Nacional dos Direitos da Pessoa com deficiência. SDHPR/SNPD, 2013. 92 p. Disponível em: $<$ http://www.pessoacomdeficiencia.gov.br/app/sites/default/files/arquivos/\%5Bfield g enerico imagens-filefield-description\%5D 0.pdf>. Acesso em: 02 abr. 2016.

. Presidência da República. Casa Civil. Subchefia para Assuntos Jurídicos. Lei № 13.146, de 6 de julho de 2015. Institui a Lei Brasileira de Inclusão da Pessoa com Deficiência. Brasília (DF). 2015. Disponível em:http://www.planalto.gov.br/ccivil 03/ Ato2015-2018/2015/Lei/L13146.htm. Acesso em: 08 set. 2017.

CAMARGO, Ana Luiza de Brasil. As dimensões e os desafios do desenvolvimento sustentável: concepções, entraves e implicações à sociedade humana. 197 fl. Dissertação (Mestrado em Engenharia de Produção). Universidade Federal de Santa Catarina- UFSC, Florianópolis, Santa Catarina, 2002.

GHANEM, Elie (Org.). Influir em políticas públicas e provocar mudanças sociais: experiências a partir da sociedade civil brasileira. São Paulo: Ashoka: Avina: Imprensa Oficial do Estado de São Paulo, 2007. 232p. Disponível em: <http://www.cedaps.org.br/wp-content/uploads/2013/07/politicas publicas.pdf> Acesso em: 08 set. 2017.

GRUPO DE ESTUDOS MULTIDISCIPLINARES DA AÇÃO AFIRMATIVA. Ações afirmativas. 2011. Disponível em: $<$ http://gemaa.iesp.ueri.br/o-que-sao-acoesafirmativas/?option=com k2\&view=item\&layout=item\&id=96\&ltemid=214 $>$. Acesso em: 11 nov. 2017

INSTITUTO BRASILEIRO DE GEOGRAFIA E ESTATÍSTICA. Indicadores de Desenvolvimento Sustentável, 2012. Disponível em: $<$ ttp://geoftp.ibge.gov.br/documentos/recursos naturais/indicadores desenvolviment o sustentavel/2012/ids2012.pdf >. Acesso em: 05 set. 2016

LENZA, Pedro. Direito constitucional esquematizado.- São Paulo : Saraiva, 2012.

MARCONI, Marina de Andrade; LAKATOS, Ana Luiza. Fundamentos de Metodologia Científica. São Paulo: Atlas S.A, 2003.

MOURA, Nághela Gonçalves de. A construção de um desenvolvimento sustentável inclusivo. 96 fl. Dissertação (Mestrado em Desenvolvimento Regional Sustentável). Universidade Federal do Cariri - UFCA, Juazeiro do Norte, Ceará, 2017.

NACIONES UNIDAS. Transformar nuestro mundo: la Agenda 2030 para el Desarrollo Sostenible. 2015. 40 p. Disponível em: $<$ http://www.un.org/ga/search/view doc.asp?symbol=A/70/L.1\&Lang=S $>$. Acesso em: 22 out. 2017 
ORGANIZAÇÃO MUNDIAL DA SAÚDE. Relatório mundial sobre a deficiência. Tradução Lexicus Serviços Linguísticos. São Paulo, 2012, 334 p. Disponível em: $<$ http://whalibdoc.who.int/publications/2011/9788564047020 por.pdf $>$. Acesso em: 10 ago. 2017.

RIBEIRO, Fernando Antônio Medeiros de Campos. O que esperar da Reunião da ONU sobre deficiência e desenvolvimento. Pauta Inclusiva. Brasília, n. 8, p. 4, jun. 2013. Disponível em:

$<$ http://www.pessoacomdeficiencia.gov.br/app/sites/default/files/arquivos/\%5Bfield g enerico imagens-filefield-description\%5D 48.pdf>. Acesso em: 15 ago. 2015

RUA, Mana das Graças; ROMANINI, Roberta. Para Aprender Políticas Públicas. Volume I: Conceitos e Teorias. Brasília: IGEPP, 2013.

SANTOS, Wederson Rufino dos. Pessoas com deficiência: nossa maior minoria. Physis - Revista de Saúde Coletiva, Rio de Janeiro, v. 18, n. 3, p. 501-510, set. 2008. Disponível em: <http://www.scielo.br/pdf/physis/v18n3/v18n3a08.pdf $>$. Acesso em: 28 ago. 2017.

SASSAKI, Romeu Kazumi. Nada sobre nós, sem nós: Da integração à inclusão Parte 1. Revista Nacional de Reabilitação, ano X, n. 57, jul./ago. 2007, p. 8-16.

SECCHI, Leonardo. Políticas públicas: conceitos, esquemas de análise, casos práticos. São Paulo: Cengage Learning, 2013.

TEIXEIRA, Marina Codo Andrade. Políticas públicas para pessoas com deficiência no Brasil. $129 \mathrm{fl}$. Dissertação (Mestrado em Administração de Empresas). Fundação Getulio Vargas - FGV, São Paulo, São Paulo, 2010.

UNITED NATIONS. Future We Want - Outcome document. 2012. Disponível em: $<$ http://sustainabledevelopment. un.org/futurewewant.html>. Acesso em: 22 out. 2017.

Werneck, Claudia. Qual a relação entre acessibilidade e sustentabilidade? Rio de Janeiro: Escola de Gente - Comunicação em Inclusão, 2011. 